\title{
When Does Compromise Prevent More Pollution?
}

\author{
C. Clemons, J. Cossey, M. Ferrara, S. Forcey, T. Norfolk, G. Obeng, \\ D. Ricciardi, and G. Young
}

\section{Introduction}

The word that economists use for a side effect of production is externality. This seemingly neutral term has earned a negative connotation. Externalities, like side effects in general, are usually unwelcome.

The discovery of a natural resource in new abundance is followed by a rapid growth in the industry of extracting it-and an equally rapid rise in pollution of the habitats nearby. There will be a cleanup cost, and it must be paid by those benefitting from the resource. That includes the firm that is polluting and the taxpayers who are happy to purchase the product at the pump or through their furnaces. Two questions are raised. It must be decided when the cost should be paid-as

C. Clemons is professor of mathematics at The University of Akron. His email address is cclemons@uakron.edu.

J. Cossey is assistant professor of mathematics at The University of Akron. His email address is cossey@uakron. edu. M. Ferrara is assistant professor of mathematics at the University of Colorado, Denver. His email address is mi chae 1 . ferrara@ucdenver.edu.

S. Forcey is assistant professor of mathematics at The University of Akron. His email address is sf34@uakron.edu.

T. Norfolk is professor of mathematics at The University of Akron. His email address is norfolk@uakron.edu.

G. Obeng is instructor of mathematics at Middle Tennessee State University. His email address is George. Obeng@mtsu . edu.

D. Ricciardi has just completed her undergraduate degree in mathematics at The University of Akron. Her email address is ricciardi@uakron.edu.

G. Young is professor of mathematics at The University of Akron. His email address is gwyoung@uakron. edu.

DOI: http://dx.doi.org/10.1090/noti893

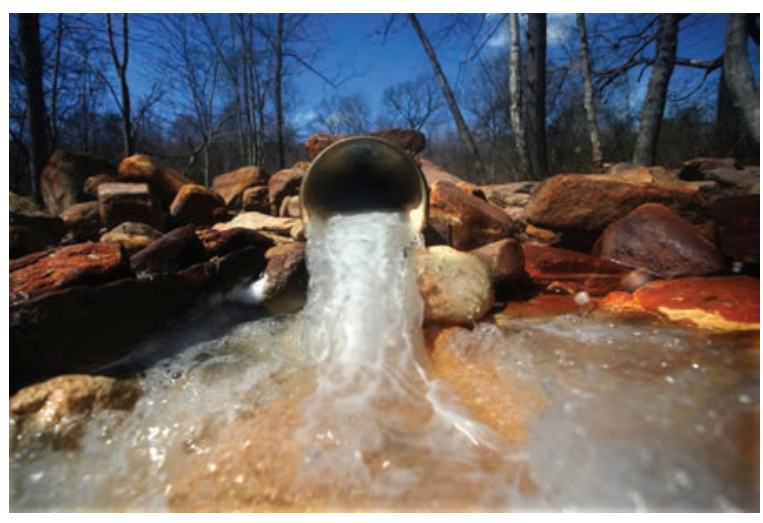

Figure 1. Treated fracking fluid headed for Black Lick Creek in Indiana County, Pa. Photo by Seamus Murphy/VII, as seen in [4].

prevention or as cure-and who should pay what percentage of each. The regulator's objective is to find a balance between public safety, economic prosperity, and tax revenues.

Using a mathematical model will always fall short when politics and psychology are major factors. Philosophical justification for studying a social problem mathematically is found in demonstrating that a solution exists. This emphasized phrase is the punch line of a joke involving fire and a mathematician with a pitcher of water, but it can also support an argument for action.

\section{Main Theorem}

By compromise, we refer to the mutual decision of government and industry to each pay for some fraction of pollution abatement. In game theory terms, compromise is a mixed equilibrium 
strategy. We prove that in certain situations there are simultaneously several Nash equilibria. For example, there are games with three simultaneous equilibria: one strategy where the firm alone pays to prevent pollution, another where only the government does, and a third in which the two players pay for precisely calculated percentages of the preventative controls. We show that the third, mixed, strategy often results in a greater amount of prevented pollution while finding a compromise between corporate profit and tax revenues. Here is a more precise statement, regarding the game we describe in Table 2 and the subsection "Proof of Theorem 1".

Theorem 1. If the product of the best-case prevention-to-cleanup cost ratios for a firm and a regulator is less than the difference of the cube and the square of the fraction of pollution prevented, then the mixed equilibrium strategy prevents more pollution than either pure equilibrium strategy. The converse is also true.

The symbolic statement of the hypothesis of this theorem is $F R<\delta^{2}-\delta^{3}$. The definitions are discussed in the subsection "The Model". The proof is found in the subsection "Proof of Theorem 1". Theorems 3 and 4 show how the hypothesis is changed when the Pigouvian tax varies in the game. There is a quite concrete implication of Theorem 1:

Corollary 2. If the product of the prevention-tocleanup cost ratios for a firm and a regulator is greater than $\frac{4}{27}$, then the mixed equilibrium strategy prevents less pollution than either pure equilibrium strategy.

\section{Assumptions and Justifications}

The chief justification for all our assumptions is this: we restrict our attention to a simple case that allows us to tractably describe the effects of policy on an economic game involving pollution.

We assume there are only two players: Player 1 is the regulator and Player 2 is the firm. Before the game the regulator decides the values of two game parameters: the corporate income tax and the percentage of expected cleanup cost to be covered by the firm, the pollution tax known as a Pigouvian tax. The regulator spends from its income tax revenue to cover the remaining percentage of cleanup costs. Cleanup costs are incurred in correcting the effects of either an accidental spill or intentional dumping of less-than-pristine wastewater into the environment. Corrective action could include habitat restoration, resettlement, covering medical bills, and compensating losses.

In the game proper both the firm and the regulator decide independently whether or not to pay for a given preventative measure, which we call the optional control of pollution. Example controls include paying for extra quality control (inspectors) or preventative technology (oil well monitoring). They might also include paying for actual cleaning technology, such as additional carbon dioxide scrubbers at a coal-burning plant or filtering of wastewater at a fracking operation. There are four possible outcomes in this nonzero sum game, and the payoff values are expected values, not random.

The firm gets to deduct the cost of optional prevention from their taxable corporate profit. The Pigouvian pollution tax however is not deductible. This latter pollution tax is assessed as a tax on production, so the question of detection of pollution is not relevant. We study the effect on the game when the rate of the pollution tax, as a percentage of cleanup covered by the firm, varies depending on whether the voluntary optional controls are applied.

The expected amount of pollution depends on the quantity of production. The profit realized by the firm is the difference between the price it can charge and the cost of producing that quantity, including the costs of pollution abatement. We assume a perfectly competitive market and a constant price per unit of production. This eliminates the possibility that the costs of pollution abatement might be simply passed on to the consumers.

Our most important assumption is that the quantity of production (per year) will remain constant throughout the game, regardless of the variations in the costs of pollution prevention and pollution cleanup to the firm. Since the cost as a function of quantity produced is almost certainly nonlinear, our assumption is only justified in situations where the practical optimum level of production is constrained away from the theoretical optimum that would maximize profit for the firm, even after the extra costs are factored in.

Thus, although the four different strategies for the firm and regulator result in four different payoff curves for the firm, that payoff is always maximized at the same right-hand limit, a production quantity called $Q_{\max }$ that is determined by technological, logistical, or legal constraints. We illustrate this in Figure 3. For instance, the amount of natural gas or oil produced by a fracking well or wells may be limited by the carrying capacity of a pipeline. Alternately, the land available for drilling might be limited, so that a company operating the wells on that land is operating at capacity but has no way to expand its operations even though theoretically it is producing less than would maximize profit. Finally, there might be a legal limit, for instance, on water usage, that 


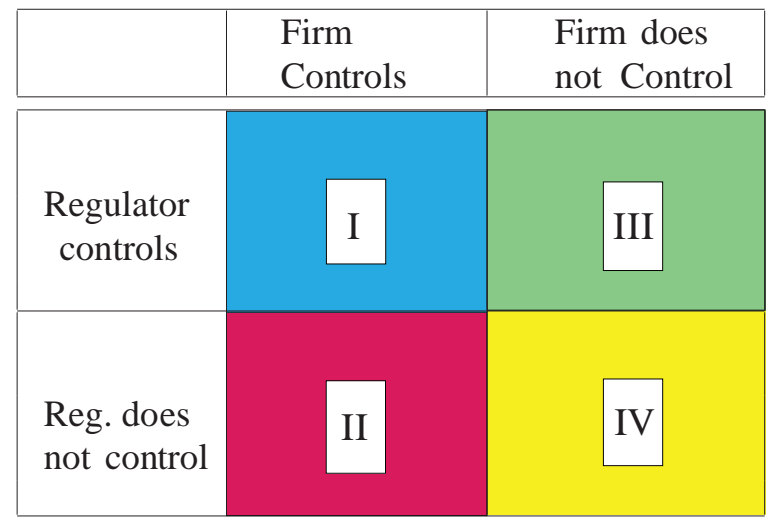

Table 1. Labels for the four game outcomes.

forces the operation to stay at or below a certain rate of production.

The goal of our analysis is to show where the Nash equilibria lie in a list of cases. A Nash equilibrium is a set of strategies (one for each of our two players) for which the payoffs are maximized for each player when the other player's strategy is held constant [6]. We assume that the choices for preventative controls made by the regulator and the firm will stabilize: they will fall into a Nash equilibrium, mixed or pure.

Our study is comparable to a snapshot, although we will mention how the decisions might be made in sequence or repeatedly over a series of years. Recent studies of similar situations have opted to simplify the number of varying factors while adding realistic complexity to their treatment of the passage of time. In [7] Tapiero considers a very similar game; to his model we add the factor $\delta$ (effectiveness of the controls) and the variable Pigouvian penalties. In [8] the same author considers the game in a queueing framework. The game is rendered tractable in that paper by deriving the model from equilibrium arguments of the queue. In [1] the authors study polluter/regulator games as stochastic processes, to be solved using control theory.

\section{The Model}

Several constant values are given at the start of the game, and several variables may be set by the regulating agency. The constant values include the profit and costs of preventative controls (for the time period determined by the application). Also, we assume there exists a probability of pollution depending on quantity of production, which is therefore a constant since production is assumed constant. This probability can be interpreted as the frequency or the likelihood of pollution, or as the fraction of total pollutant released. Either way, the probability $p$ multiplied by the cost of pollution $\Delta$ can be interpreted as the expected cost of cleanup for the time period. Finally, there is the fraction $\delta$ of cleanup costs prevented by a set of optional controls: we assume it to be the same whether those controls are implemented by the firm or by the regulator.

The constants are:

$P_{F} \quad \ldots \quad$ the profit being achieved by the firm, before tax and before paying for any voluntary preventative controls and mandatory cleanup costs,

$C_{F} \quad \ldots \quad$ the cost to the firm of optional control,

$C_{R} \quad \ldots \quad$ the cost to the regulator of optional control,

$p \Delta \quad \ldots \quad$ the total expected cleanup costs,

$\delta \quad \ldots \quad$ the effectiveness of the optional controls,

obeying: $P_{F}>0,0 \leq p \leq 1$, and $0 \leq \delta \leq 1$.

Thus if either just the firm or just the regulator applies optional controls, the remaining expected cost of cleanup is $(1-\delta) p \Delta$. If both sets of optional controls are applied then the cost of cleanup is decreased again, leaving the remaining cost of cleanup, $(1-\delta)^{2} p \Delta$. A possible future study might introduce two different strengths, $\delta_{f}$ and $\delta_{r}$, for two different controls purchased by the firm and by the regulator. Here, though, we are considering only one sort of control at a time. Thus the regulator's purchase can be seen as a subsidy of pollution control actually performed by the firm. That also explains why we consider two separate costs of control: the regulator may not pay the same for a filter as would a private firm.

The variables determined by the regulator (via its policymaking body) are the tax rate applied to the firm's profit and the variable percentages of cleanup cost covered by the regulator. Tax is assessed before any cleanup costs incurred by the firm but after any optional preventative control costs are subtracted from the profits.

$\tau$
$\quad \ldots$ the corporate tax rate on profit minus any cost of controls,

$\alpha_{1} \quad \ldots \quad$ the percentage of cleanup costs paid by the regulator if the firm opts for extra controls,

$\alpha_{3} \quad \ldots \quad$ the percentage of cleanup costs paid by the regulator when the firm does not apply extra controls but the regulator does,

$\alpha_{4} \quad \ldots \quad$ the percentage of cleanup costs paid by the regulator if neither firm nor regulator opt for extra controls.

For instance, if neither the regulator nor the firm adopts the optional controls, then the cost of cleanup for the regulator is $\alpha_{4} p \Delta$ and the cost of cleanup to the firm is $\left(1-\alpha_{4}\right) p \Delta$. If only the 
Table 2. The payoff matrix: Firm's payoff is above regulator's in each cell.

\begin{tabular}{|c|c|c|}
\hline & Firm controls & Firm does not control $(1-y)$ \\
\hline \multirow{2}{*}{$\begin{array}{l}\text { Regulator } \\
\text { controls }(x)\end{array}$} & $\begin{array}{c}f_{1}=(1-\tau)\left(P_{F}-C_{F}\right) \\
\quad-(1-\delta)^{2} p \Delta\left(1-\alpha_{1}\right) \\
\end{array}$ & $f_{3}=(1-\tau) P_{F}-(1-\delta) p \Delta\left(1-\alpha_{3}\right)$ \\
\hline & $\begin{aligned} r_{1}= & \tau\left(P_{F}-C_{F}\right)-C_{R} \\
& -(1-\delta)^{2} p \Delta \alpha_{1}\end{aligned}$ & $r_{3}=\tau P_{F}-C_{R}-(1-\delta) p \Delta \alpha_{3}$ \\
\hline \multirow{2}{*}{$\begin{array}{l}\text { Reg. does } \\
\text { not control } \\
\quad(1-x)\end{array}$} & $\begin{array}{c}f_{2}=(1-\tau)\left(P_{F}-C_{F}\right) \\
-(1-\delta) p \Delta\left(1-\alpha_{1}\right)\end{array}$ & $f_{4}=(1-\tau) P_{F}-p \Delta\left(1-\alpha_{4}\right)$ \\
\hline & $\begin{array}{l}r_{2}=\tau\left(P_{F}-C_{F}\right) \\
\quad-(1-\delta) p \Delta \alpha_{1}\end{array}$ & $r_{4}=\tau P_{F}-p \Delta \alpha_{4}$ \\
\hline
\end{tabular}

firm adopts the extra controls, then the cost of cleanup to the regulator is $\alpha_{1}(1-\delta) p \Delta$.

The subscripts used on the variable $\alpha$ correspond to outcomes (labeled by corresponding Roman numerals) in the $2 \times 2$ game shown in Table 1 . The subscript 2 is not used, since we assume that if the firm adopts controls then it is guaranteed a single percentage $\alpha_{1}$. The percentages $\left(1-\alpha_{i}\right)$ multiplied by the expected cost of cleanup can be interpreted as the penalties paid by the firm for polluting. Here we assume that $0 \leq \alpha_{i} \leq 1$, but an easy extension would allow negative values of $\alpha_{i}$ to indicate penalties beyond the cost of cleanup.

The payoff for the firm is the amount of after-tax profit remaining after paying for cleanup and/or prevention. Similarly, the payoff for the regulator is the amount of tax revenue retained. Table 2 shows the payoff for the firm above the payoff for the regulator in each of the four outcomes from Table 1.

We have also introduced new variables in Table 2. These will be useful in describing mixed strategies. If pollution is being filtered, $x$ and $y$ are the proportions of the full strength of the filtering mechanism. That is, the regulator purchases a filter that has only fraction $x$ of effectiveness $\delta$, and the firm purchases a filter of fractional strength $y \delta$. Alternatively, in a series of time periods, perhaps multiple years, the variable $x$ could be the proportion of the years for which the regulator adopts preventative controls, while the variable $y$ is the proportion of the years for which the firm controls. Or, if there are a number of extraction wells (or mines or power plants), $x$ and $y$ may also be interpreted as the proportions of facilities with extra controls applied by the regulator or firm respectively.

We will assume that the controls, such as a pair of filters of fractional strengths $x$ and $y$, are used by the firm in a coordinated fashion. Thus the expected fraction (frequency) of the pollution released into the environment is given by

$$
(1-x \delta)(1-y \delta) p .
$$

Notice that this expression is derived either by considering the remaining fraction of pollution after successively subtracting the two fractions $x \delta$ and $y \delta$ or alternatively by applying $x$ and $y$ to the respective amounts of pollution in Table 2, as in the calculation of the mixed payoffs.

\section{Analyzing the Game Penalties}

The following analysis will be organized into sections comparing the results for several ways of choosing values for $\alpha_{i}$. In each we will compare the pure and mixed strategies. The cases we consider are as follows:
(1) $\alpha_{1}=\alpha_{3}=\alpha_{4}$,
(2) $\alpha_{1}=\alpha_{3}>\alpha_{4}$,
(3) $\alpha_{1}=\alpha_{3}<\alpha_{4}$,
(4) $\alpha_{1}>\alpha_{3}=\alpha_{4}$.

Since $\alpha_{i}$ is the fraction of cleanup covered by the regulator, a smaller value of this fraction can be considered a contingent penalty levied on the firm. In case (1) the penalty is uniform: there is no contingent portion of the penalty based on the optional controls. In case (2) the contingent penalty is only applied if neither the firm nor the regulator pays for extra controls. Here the regulator rather generously waives the penalty on the firm for not controlling as long as the regulator pays for the additional controls itself. Case (3) is the reverse situation: the contingent penalty is waived only when neither the firm nor the regulator pays for extra controls. Finally, in case (4) the contingent penalty is applied whenever the firm opts not to take the extra preventative measures. 


\section{Commonalities}

Before considering these cases we perform the analysis common to all of them. First we define two new quantities from the given constants. Both quantities are defined using the fraction $\alpha_{1}$. Recall that $\alpha_{1}$ gives the fraction of cost of cleanup paid by the regulator in the best-case scenario, when both parties simultaneously pay for extra prevention. We can refer to the following ratios as best-case cost ratios. By $F$ we denote the ratio of control cost to cleanup cost for the firm, and by $R$ the same ratio for the regulator:

$$
F=\frac{(1-\tau) C_{F}}{\left(1-\alpha_{1}\right) p \Delta} \text { and } R=\frac{C_{R}}{\alpha_{1} p \Delta} .
$$

Pure Equilibria. A pure equilibrium is when one of the four cells in the game describes a Nash equilibrium, that is, when $x, y \in\{0,1\}$. Pure Nash equilibrium strategies exist in the regions of Table 1 when the following inequalities hold:

$$
\text { I } f_{1} \geq f_{3} \text { and } r_{1} \geq r_{2} \text {. Thus }
$$

$$
F \leq(1-\delta) \frac{1-\alpha_{3}}{1-\alpha_{1}}-(1-\delta)^{2} \text { and } R \leq \delta-\delta^{2} \text {. }
$$

II $f_{2} \geq f_{4}$ and $r_{2} \geq r_{1}$. Thus

$$
F \leq \frac{1-\alpha_{4}}{1-\alpha_{1}}-1+\delta \text { and } R \geq \delta-\delta^{2} .
$$

III $f_{3} \geq f_{1}$ and $r_{3} \geq r_{4}$. Thus

$F \geq(1-\delta) \frac{1-\alpha_{3}}{1-\alpha_{1}}-(1-\delta)^{2}$ and $R \leq \frac{\alpha_{4}}{\alpha_{1}}-(1-\delta) \frac{\alpha_{3}}{\alpha_{1}}$.

IV $f_{4} \geq f_{2}$ and $r_{4} \geq r_{3}$. Thus

$$
F \geq \frac{1-\alpha_{4}}{1-\alpha_{1}}-1+\delta \text { and } R \geq \frac{\alpha_{4}}{\alpha_{1}}-(1-\delta) \frac{\alpha_{3}}{\alpha_{1}} .
$$

Mixed Equilibria. Whether or not a pure strategy exists, there may also be mixed strategies that represent equilibria. If the firm controls $y$ percent of the time and the regulator controls $x$ percent of the time, then their respective payoffs are

$f_{m}=y\left(x f_{1}+(1-x) f_{2}\right)+(1-y)\left(x f_{3}+(1-x) f_{4}\right)$, $r_{m}=x\left(y r_{1}+(1-y) r_{3}\right)+(1-x)\left(y r_{2}+(1-y) r_{4}\right)$.

A Nash equilibrium occurs when the firm and regulator choose percentages $x$ and $y$ such that neither could get a better payoff by unilaterally changing its choice. That means $f_{m}$ would be at a local max in the $y$ direction and $r_{m}$ would be at a local max in the $x$ direction. So we set partial derivatives equal to zero: $\partial_{y} f_{m}=0$ and $\partial_{x} r_{m}=0$. Solving yields

$$
\begin{gathered}
x=\left(f_{4}-f_{2}\right) /\left(f_{1}-f_{2}-f_{3}+f_{4}\right) \\
\text { and } \\
y=\left(r_{4}-r_{3}\right) /\left(r_{1}-r_{3}-r_{2}+r_{4}\right) .
\end{gathered}
$$

Thus, via simplifying and writing in terms of $F$ and $R$ :

$$
\begin{gathered}
\quad x= \\
\frac{(1-\delta)\left(1-\alpha_{1}\right)-\left(1-\alpha_{4}\right)+(1-\tau) C_{F} / p \Delta}{(1-\delta)\left(1-\alpha_{1}\right)-\left(1-\alpha_{4}\right)+(1-\delta)\left(1-\alpha_{3}\right)-(1-\delta)^{2}\left(1-\alpha_{1}\right)} \\
=\frac{F+1-\delta-\frac{1-\alpha_{4}}{1-\alpha_{1}}}{\delta-\delta^{2}+(1-\delta) \frac{1-\alpha_{3}}{1-\alpha_{1}}-\frac{1-\alpha_{4}}{1-\alpha_{1}}}
\end{gathered}
$$

and

$$
\begin{aligned}
y & =\frac{(1-\delta) \alpha_{3}-\alpha_{4}+C_{R} / p \Delta}{(1-\delta) \alpha_{3}-\alpha_{4}+\left(\delta-\delta^{2}\right) \alpha_{1}} \\
& =\frac{R+(1-\delta) \frac{\alpha_{3}}{\alpha_{1}}-\frac{\alpha_{4}}{\alpha_{1}}}{\delta-\delta^{2}+(1-\delta) \frac{\alpha_{3}}{\alpha_{1}}-\frac{\alpha_{4}}{\alpha_{1}}} .
\end{aligned}
$$

Remark 1. In theory the rates $\tau$ and $\alpha_{i}$ could be set to ensure an equilibrium in which both players prevent additional pollution or to avoid the situation in which neither do. Realistically, the cost to the overall economy might be too high. There will often be a mixed strategy. Here policy might be set in order to maximize both $x$ and $y$ (as close to 1 as possible). Ideally then we set $y=1$ and find

$$
\alpha_{1}=\frac{C_{R}}{\left(\delta-\delta^{2}\right) p \Delta} .
$$

Then setting $x=1$ leads to

$$
\tau=1-\frac{(1-\delta)\left(1-\alpha_{3}\right)-(1-\delta)^{2}\left(1-\alpha_{1}\right)}{C_{F} / p \Delta} .
$$

\section{Case 1}

We begin with the "base" case, in which the percentage of responsibilities for cleanup are always the same: $\alpha_{1}=\alpha_{3}=\alpha_{4}$.

Now the pure equilibria, using the labels from Table 1, occur when:
I. $F \leq \delta-\delta^{2}$ and $R \leq \delta-\delta^{2}$,
II. $F \leq \delta$ and $R \geq \delta-\delta^{2}$,
III. $F \geq \delta-\delta^{2}$ and $R \leq \delta$,
IV. $F \geq \delta$ and $R \geq \delta$.

Notice that if outcome I is an equilibrium, then $F \leq F+\delta^{2} \leq \delta$ and $R \leq R+\delta^{2} \leq \delta$. Thus $f_{1} \geq f_{3}$ and $f_{2} \geq f_{4}$, showing that controlling for pollution is a dominant strategy for the firm and the same is true for the regulator. Similarly, if outcome IV is an equilibrium, then not controlling pollution is dominant for both players.

For the mixed strategies, the requirement that $x, y$ are both nonnegative fractions of 1 implies that $\delta-\delta^{2} \leq F \leq \delta$ and that $\delta-\delta^{2} \leq R \leq \delta$.

We can illustrate these equilibria as regions on a graph, where the axes are labeled by $F$ and $R$. Figure 2 shows the results.

Remark 2. If our ideal is minimizing pollution, then we look at how we might achieve equilibrium either in region I (a pure strategy) or in a 


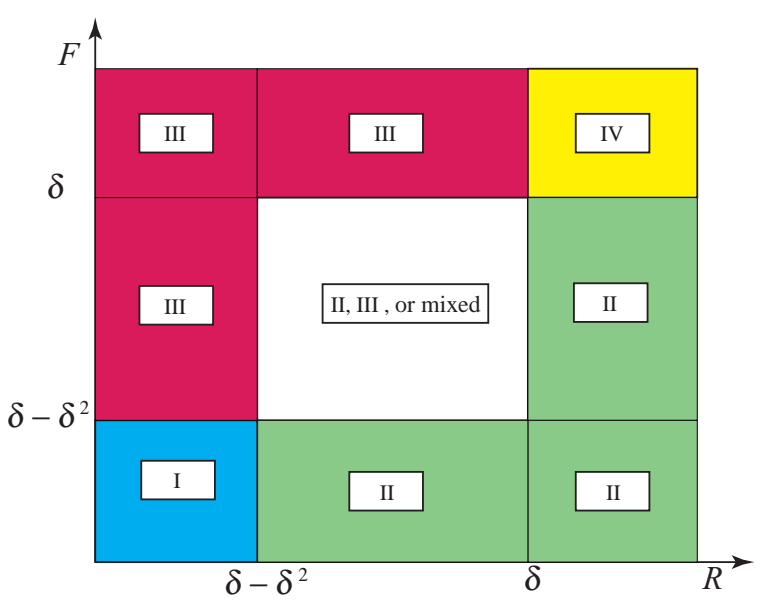

Figure 2. Nash equilibria relative to $F$ and $R$ in

Case 1.

mixed strategy as close as possible to it. Hence we choose

$$
\alpha_{1}=\frac{C_{R}}{\left(\delta-\delta^{2}\right) p \Delta}
$$

and then

$$
\tau=1-\frac{\left(\delta-\delta^{2}\right) p \Delta-C_{R}}{C_{F}} .
$$

Using these approximate values for $\alpha_{1}$ and $\tau$ in the definitions of $F$ and $R$ gives

$$
F R=\delta^{2}-\delta^{3}-\left(\delta^{3}-\delta^{4}\right)<\delta^{2}-\delta^{3} .
$$

This inequality is the hypothesis of Theorem 1 , which is expected, since maximizing $x$ and $y$ should also maximize pollution prevention.

Example 1. We look at a simple example to illustrate several of the facts just described:

$$
\begin{array}{ll}
\text { Given } & \text { Calculated } \\
P_{F}=1000 & F=0.192 \\
\Delta=500 & R=0.32 \\
C_{F}=40 & \delta-\delta^{2}=0.1875 \\
C_{R}=40 & x=0.992 \\
\alpha_{1}=\alpha_{3}=\alpha_{4}=0.5 & y=0.764 \\
\tau=0.4 & f_{m}=568 \\
\delta=0.75 & r_{m}=334.436 \\
p=0.5 &
\end{array}
$$

Figure 3 shows a hypothetical pretax profit curve and four hypothetical payoff curves for the firm. Over the constraining quantity $Q_{\max }$ we find the four payoff values that are calculated using the formulas from Table 2. Since $F$ and $R$ lie between $\delta$ and $\delta-\delta^{2}$, we predict three equilibria, two pure and one mixed, in the resulting game:

\begin{tabular}{|c|c|c|}
\hline Example 1. & Firm controls & Firm does not control \\
\hline \hline $\begin{array}{c}\text { Reg. } \\
\text { controls }\end{array}$ & $f_{1}=568.1875$ & $f_{3}=568.75$ \\
& $r_{1}-336.1875$ & $r_{3}-328.75$ \\
\hline $\begin{array}{c}\text { Reg. does } \\
\text { not control }\end{array}$ & $f_{2}=544.75$ & $f_{4}=475$ \\
& $r_{2}-352.75$ & $r_{4}-275$ \\
\hline
\end{tabular}

The two pure equilibria are as predicted in outcomes II and III, and the mixed equilibrium is found at $x=0.992$ and $y=0.764$. The two pure equilibria both result in a new frequency of pollution given by $(1-\delta) p=0.125$. The mixed strategy, however, gives a new frequency of pollution, $(1-x \delta)(1-y \delta) p=0.0546$. Thus the mixed strategy has better than halved the expected amount of pollution from either pure strategy. At the same time, the payoff for the regulator in the mixed strategy is $r_{m}=334.436$, better than one of the equilibria strategies. The firm is happy about the mixed strategy, since its payoff is $f_{m}=568$, only slightly less than in its best-case pure equilibrium strategy.

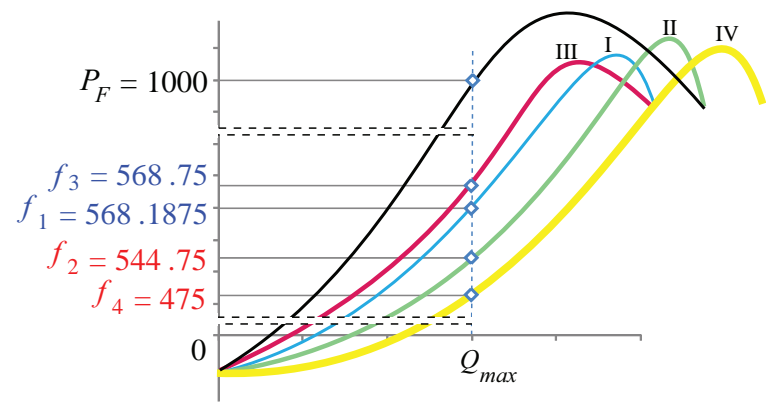

Figure 3. A hypothetical profit curve and four payoff curves depending on the strategies chosen in the game of our first example. The cost functions are unknown, but our assumption is that the maximum payoffs are found at a constraining limit on production.

Proof of Theorem 1. The preceding example leads us to investigate the question of when, in general, the mixed strategy turns out to prevent more pollution than either pure strategy (where just one player controls the pollution). This happens when

$$
(1-x \delta)(1-y \delta) p<(1-\delta) p,
$$

which reduces quickly to

$$
x y \delta<x+y-1 .
$$


In the current case, when no extra penalties depend on the chosen strategy, we have

$$
x=\frac{\delta-F}{\delta^{2}} \quad \text { and } \quad y=\frac{\delta-R}{\delta^{2}} .
$$

Then the condition becomes

$$
F R<\delta^{2}-\delta^{3},
$$

which is the proof of Theorem 1 .

In the preceding example, $\delta^{2}-\delta^{3}=.1406$ and $F R=0.0614$, illustrating the predictive power of the theorem.

The corollary to Theorem 1 states that if $F R>\frac{4}{27}$, then the mixed strategy prevents less pollution than the pure ones. This is seen by noting that the maximum value of $\delta^{2}-\delta^{3}$ for $0 \leq \delta \leq 1$ occurs at $\delta=\frac{2}{3}$. In Figure 4 we see the entire curve.

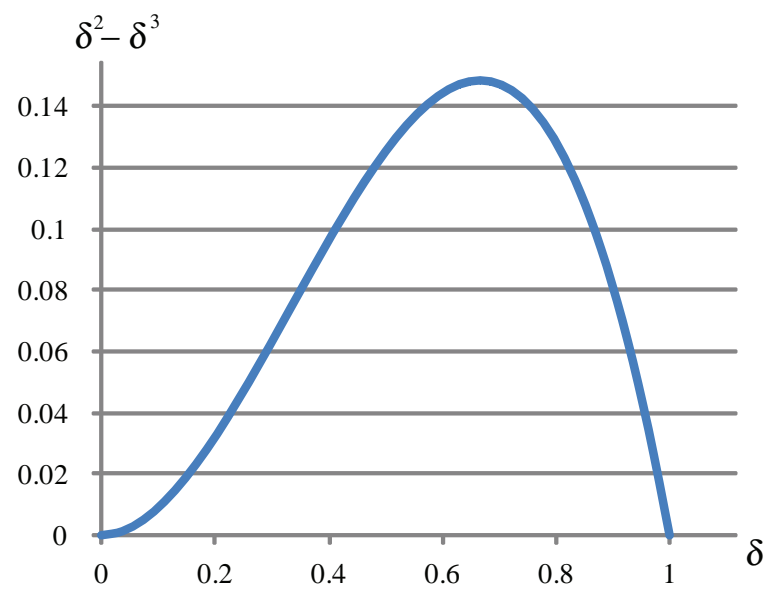

Figure 4. The maximum of this curve is $\frac{4}{27}$.

\section{Case 2}

Here the penalty on the firm is waived if the firm does not control but the regulator does. This might seem counterintuitive, but it may be coupled with higher taxes. When $\alpha_{1}=\alpha_{3}>\alpha_{4}$, the pure equilibria, again using the labels from Table 1, occur when

$$
\begin{aligned}
& \text { I. } F \leq \delta-\delta^{2} \text { and } R \leq \delta-\delta^{2} \\
& \text { II. } F \leq \delta+\frac{\alpha_{1}-\alpha_{4}}{1-\alpha_{1}} \text { and } R \geq \delta-\delta^{2}, \\
& \text { III. } F \geq \delta-\delta^{2} \text { and } R \leq \delta-\left(1-\frac{\alpha_{4}}{\alpha_{1}}\right) \\
& \text { IV. } F \geq \delta+\frac{\alpha_{1}-\alpha_{4}}{1-\alpha_{1}} \text { and } R \geq \delta-\left(1-\frac{\alpha_{4}}{\alpha_{1}}\right) .
\end{aligned}
$$

For the mixed strategies, the requirement that $x$ be a nonnegative fraction of 1 implies that

$$
\delta-\delta^{2} \leq F \leq \delta+\frac{\alpha_{1}-\alpha_{4}}{1-\alpha_{1}} .
$$

The requirement that $y$ be a nonnegative fraction of 1 leads to two subcases: (2a) If $1-\frac{\alpha_{4}}{\alpha_{1}}<\delta^{2}$, then

$$
\delta-\delta^{2} \leq R \leq \delta-\left(1-\frac{\alpha_{4}}{\alpha_{1}}\right) .
$$

(2b) If $1-\frac{\alpha_{4}}{\alpha_{1}}>\delta^{2}$, then

$$
\delta-\left(1-\frac{\alpha_{4}}{\alpha_{1}}\right) \leq R \leq \delta-\delta^{2} .
$$

Figures 5 and 6 show the results for subcases (2a) and $(2 \mathrm{~b})$ respectively.

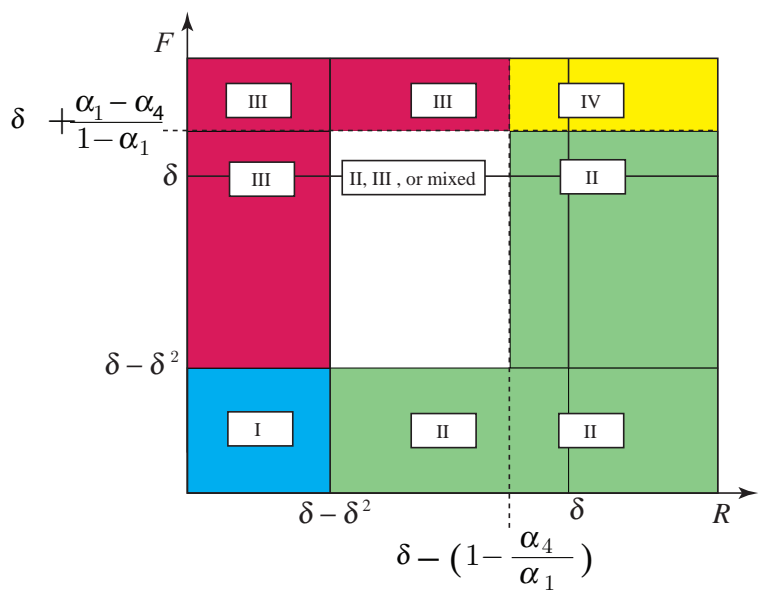

Figure 5. Results of subcase (2a), when $1-\frac{\alpha_{4}}{\alpha_{1}}<\delta^{2}$.

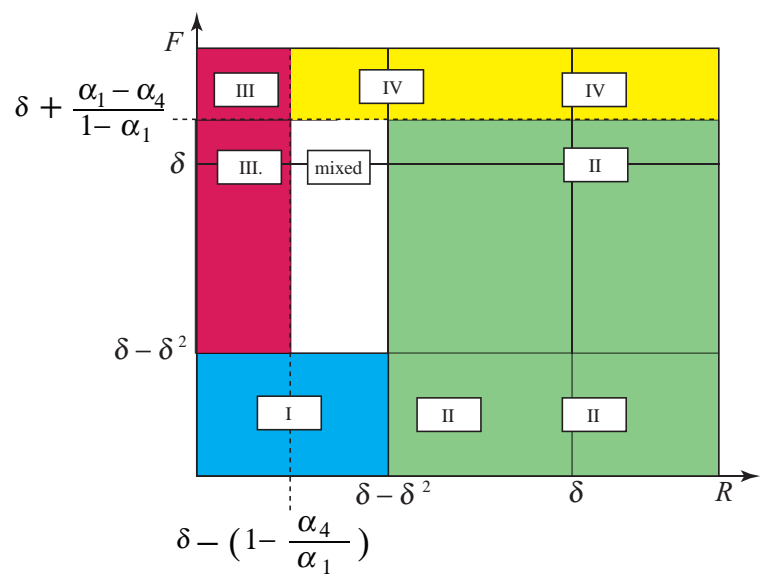

Figure 6. Results of subcase ( $2 b)$, when $1-\frac{\alpha_{4}}{\alpha_{1}}>\delta^{2}$.

Remark 3. In both figures showing Case 2 we see that the region marked II has increased in area relative to the region marked II in Case 1. We can argue qualitatively from these figures that all else being equal, the situation in which the firm is the only controller is more likely to occur in Case 2.

Since subcase (2a) has the possibility of three equilibria, we again consider what conditions guarantee that the mixed equilibrium will result in greater pollution abatement. 
Theorem 3. In subcase (2a), the mixed equilibrium will result in greater pollution abatement if and only if

$$
\begin{gathered}
F R+\frac{1-\delta}{\alpha_{1}}\left(\frac{\alpha_{1}-\alpha_{4}}{1-\alpha_{1}}\right) \\
\times\left(\alpha_{1}-\alpha_{4}+\delta-2 \alpha_{1} \delta+\alpha_{1} R-\left(1-\alpha_{1}\right) F\right) \\
<\delta^{2}-\delta^{3} .
\end{gathered}
$$

Proof. As in Theorem 1 the mixed equilibrium prevents more pollution when $x y \delta<x+y-1$. Here we use the conditions of subcase (2a) and do some algebraic reducing. The simplification was first performed by hand and then checked by computer.

Example 2. We illustrate the first subcase (2a) with another simple example.

$$
\begin{array}{ll}
\text { Given } & \text { Calculated } \\
P_{F}=1000 & F=0.2479 \\
\Delta=250 & R=0.2478 \\
C_{F}=55 & \delta-\delta^{2}=0.45-0.2025 \\
& \multicolumn{1}{c}{=0.2475} \\
C_{R}=7 & x=0.998 \\
\alpha_{1}=\alpha_{3}=0.226 & y=0.997 \\
\alpha_{4}=0.2 & 1-\frac{\alpha_{4}}{\alpha_{1}}=0.115 \\
\tau=0.564 & \delta-\left(1-\frac{\alpha_{4}}{\alpha_{1}}\right)=0.335 \\
\delta=0.45 & f_{m}=382.717 \\
p=0.5 & r_{m}=517.513
\end{array}
$$

Here the values of $F$ and $R$ fall in the range for which there are three equilibria. Within this example we have decided to also minimize pollution. To do so, we have calculated and used the near-optimal values

$$
\alpha_{1} \sim \frac{C_{R}}{\left(\delta-\delta^{2}\right) p \Delta} \sim 0.226
$$

and

$$
\tau \sim 1-\frac{\left(\delta-\delta^{2}\right) p \Delta-C_{R}}{C_{F}} \sim 0.565 .
$$

These choices for $\tau$ and $\alpha_{1}$ ensure that $x$ and $y$ are close to 1 . Here is the game:

\begin{tabular}{|c|c|c|}
\hline Example 2. & Firm controls & Firm does not control \\
\hline \hline $\begin{array}{c}\text { Reg. } \\
\text { controls }\end{array}$ & $f_{1}=382.75$ & $f_{3}=382.7875$ \\
\hline $\begin{array}{c}\text { Reg. does } \\
\text { not control }\end{array}$ & $r_{1}-517.434$ & $r_{3}-541.4625$ \\
& $f_{2}=517.442$ & $f_{4}=336$ \\
\hline
\end{tabular}

As predicted, there are three equilibria, including the two pure strategies at II and III. The inequality in Theorem 3 holds in this example: filling in the given values gives $0.0863<0.1114$. That fact predicts the next: the two pure equilibria both result in a new frequency of pollution given by $(1-\delta) p=0.275$, while the mixed strategy gives the much lower frequency of pollution $(1-x \delta)(1-$ $y \delta) p=0.152$.

Example 3. We return to a variation of our simple example to illustrate the second subcase (2b).

$$
\begin{array}{ll}
\text { Given } & \text { Calculated } \\
P_{F}=1000 & F=0.44 \\
\Delta=250 & R=0.187 \\
C_{F}=55 & \delta-\delta^{2}=0.45-0.2025 \\
& \multicolumn{1}{c}{=0.2475} \\
C_{R}=7 & x=0.443 \\
\alpha_{1}=\alpha_{3}=0.3 & y=0.535 \\
\alpha_{4}=0.2 & 1-\frac{\alpha_{4}}{\alpha_{1}}=0.33 \\
\tau=0.3 & \delta-\left(1-\frac{\alpha_{4}}{\alpha_{1}}\right)=0.1167 \\
\delta=0.45 & f_{m}=622.96 \\
p=0.5 & r_{m}=268.5
\end{array}
$$

Since $F$ lies between $\delta$ and $\delta-\delta^{2}$ and $R$ between $\delta-\left(1-\frac{\alpha_{4}}{\alpha_{1}}\right)$ and $\delta-\delta^{2}$, we predict only one mixed equilibrium:

\begin{tabular}{|c|c|c|}
\hline Example 3. & Firm controls & Firm does not control \\
\hline \hline $\begin{array}{c}\text { Reg. } \\
\text { controls }\end{array}$ & $f_{1}=635.03$ & $f_{3}=651.875$ \\
& $r_{1}-265.156$ & $r_{3}-272.375$ \\
\hline $\begin{array}{c}\text { Reg. does } \\
\text { not control }\end{array}$ & $f_{2}=613.375$ & $f_{4}=600$ \\
& $r_{2}-262.875$ & $r_{4}-275$ \\
\hline
\end{tabular}

As expected, here there is only the mixed equilibrium.

\section{Case 3}

Here a penalty on the firm is applied whenever either the regulator or the firm is paying for extra controls. This is really counterintuitive, and we include it for contrast. If $\alpha_{1}=\alpha_{3}<\alpha_{4}$, then pure equilibria occur just as in Case 2 when

I. $F \leq \delta-\delta^{2}$ and $R \leq \delta-\delta^{2}$,

II. $F \leq \delta+\frac{\alpha_{1}-\alpha_{4}}{1-\alpha_{1}}$ and $R \geq \delta-\delta^{2}$,

III. $F \geq \delta-\delta^{2}$ and $R \leq \delta-\left(1-\frac{\alpha_{4}}{\alpha_{1}}\right)$,

IV. $F \geq \delta+\frac{\alpha_{1}-\alpha_{4}}{1-\alpha_{1}}$ and $R \geq \delta-\left(1-\frac{\alpha_{4}}{\alpha_{1}}\right)$.

For the mixed strategies, the requirement that $y$ be a nonnegative fraction of 1 implies that

$$
\delta-\delta^{2} \leq R \leq \delta-\left(1-\frac{\alpha_{4}}{\alpha_{1}}\right) .
$$

The requirement that $x$ be a nonnegative fraction of 1 leads to two subcases:

(3a) If $\frac{\alpha_{4}-\alpha_{1}}{1-\alpha_{1}}<\delta^{2}$, then

$$
\delta-\delta^{2} \leq F \leq \delta-\frac{\alpha_{4}-\alpha_{1}}{1-\alpha_{1}} .
$$


(3b) If $\frac{\alpha_{4}-\alpha_{1}}{1-\alpha_{1}}>\delta^{2}$, then

$$
\delta-\frac{\alpha_{4}-\alpha_{1}}{1-\alpha_{1}} \leq F \leq \delta-\delta^{2} .
$$

Figures 7 and 8 show the results for subcases (3a) and $(3 \mathrm{~b})$ respectively.

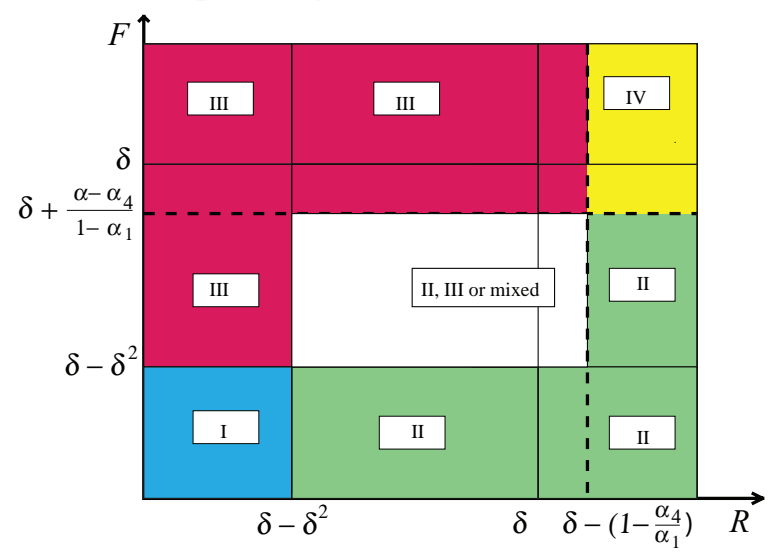

Figure 7. Results of subcase (3a).

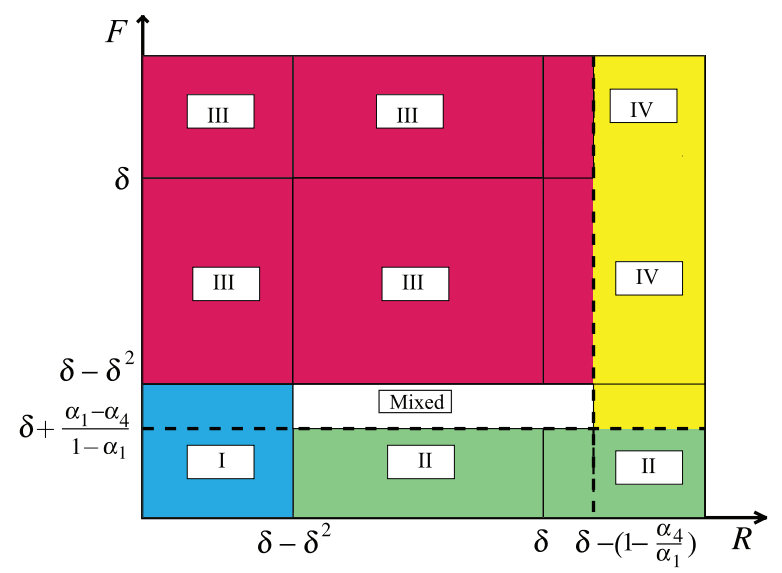

Figure 8. Results of subcase (3b).

Remark 4. In both figures showing Case 3 we see that the region marked III has increased in area relative to the region marked III in Case 1 . We can argue qualitatively from these figures that all else being equal, the situation in which the regulator is the only controller is more likely to occur in Case 3.

\section{Case 4}

Common sense leads us to the case in which a penalty is applied whenever the firm fails to implement optional controls. Thus $\alpha_{1}>\alpha_{3}=\alpha_{4}$. To simplify the notation we define

$$
\beta=\frac{\alpha_{1}-\alpha_{4}}{1-\alpha_{1}}(1-\delta)-\delta^{2}
$$

Now the pure equilibria are
I. $F \leq \delta+\beta$ and $R \leq \delta-\delta^{2}$,
II. $F \leq \delta+\frac{\alpha_{1}-\alpha_{4}}{1-\alpha_{1}}$ and $R \geq \delta-\delta^{2}$,

III. $F \geq \delta+\beta$ and $R \leq \delta \frac{\alpha_{4}}{\alpha_{1}}$,

IV. $F \geq \delta+\frac{\alpha_{1}-\alpha_{4}}{1-\alpha_{1}}$ and $R \geq \delta \frac{\alpha_{4}}{\alpha_{1}}$.

For the mixed strategies, the requirement that $x$ be a nonnegative fraction of 1 implies that

$$
\delta+\beta \leq F \leq \delta+\frac{\alpha_{1}-\alpha_{4}}{1-\alpha_{1}} .
$$

The requirement that $y$ be a nonnegative fraction of 1 leads to two subcases:

(4a) If $1-\frac{\alpha_{4}}{\alpha_{1}}<\delta$, then

$$
\delta-\delta^{2} \leq R \leq \delta \frac{\alpha_{4}}{\alpha_{1}} .
$$

(4b) If $1-\frac{\alpha_{4}}{\alpha_{1}}>\delta$, then

$$
\delta \frac{\alpha_{4}}{\alpha_{1}} \leq R \leq \delta-\delta^{2} .
$$

Since subcase (4a) has the possibility of three equilibria, we again consider what conditions guarantee that the mixed equilibrium will result in greater pollution abatement.

Theorem 4. In subcase (4a), the mixed equilibrium will result in greater pollution abatement if and only if

$$
\begin{gathered}
F R+\frac{1-\delta}{\alpha_{1}}\left(\frac{\alpha_{1}-\alpha_{4}}{1-\alpha_{1}}\right) \\
\times\left(\alpha_{1}-\alpha_{4}+\delta-2 \alpha_{1} \delta-\left(1-\alpha_{1}\right) F\right) \\
<\delta^{2}-\delta^{3} .
\end{gathered}
$$

Proof. As in Theorem 1, the mixed equilibrium prevents more pollution when $x y \delta<x+y-1$. Here we use the conditions of subcase (4a) and do some algebraic reducing. The simplification was first performed by hand and then checked by computer.

Figures 9, 10, 11, and 12 show the results for subcases (4a) and (4b) with $\beta>0$ and $\beta<0$ respectively.

Remark 5. In all figures showing subcase 4 we see that the regions marked I and II have increased in area relative to the similarly marked regions for Case 1 . We can argue qualitatively from these figures that all else being equal, the situation in which the firm is the only controller is more likely to occur in Case 4. Even more importantly, the optimal situation in which both players control pollution is most likely in Case 4 . 


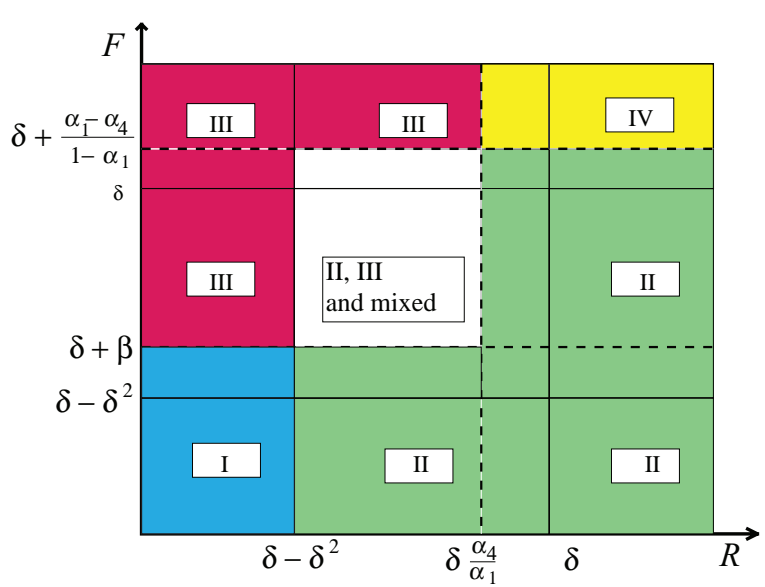

Figure 9. Results of subcase (4a), $\beta>0$.

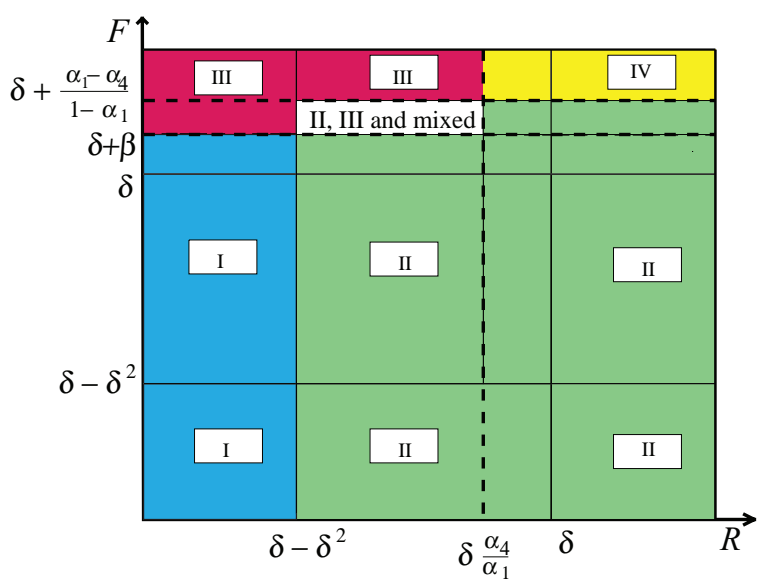

Figure 10. Results of subcase (4a), $\beta<0$.

Example 4. Here is an example that illustrates subcase $4 \mathrm{a}$ with $\boldsymbol{\beta}<\mathbf{0}$ and with three equilibria:

$\begin{array}{lll}\text { Given } & \text { Calculated } & \\ P_{F}=1000 & F=0.384 & \beta=-0.4625 \\ \Delta=500 & R=0.24 & \delta+\beta=0.2875 \\ C_{F}=80 & & \delta-\delta^{2}=0.1875 \\ C_{R}=30 & x=0.888 & \\ \alpha_{1}=0.5 & y=0.8 & \delta \frac{\alpha_{3}}{\alpha_{1}}=.45 \\ \alpha_{3}=\alpha_{4} & f_{m}=541.565 & \\ \quad=0.3 & & \\ T=0.4 & r_{m}=334.4 & \delta+\frac{\alpha_{1}-\alpha_{3}}{1-\alpha_{1}}=1.15 \\ \delta=0.75 & & \\ p=0.5 & & \end{array}$

\begin{tabular}{|c|c|c|}
\hline Example 4. & Firm controls & Firm does not control \\
\hline \hline $\begin{array}{c}\text { Reg. } \\
\text { controls }\end{array}$ & $f_{1}=544.1875$ & $f_{3}=556.25$ \\
& $r_{1}-330.1875$ & $r_{3}-351.25$ \\
\hline $\begin{array}{c}\text { Reg. does } \\
\text { not control }\end{array}$ & $f_{2}=520.75$ & $f_{4}=425$ \\
& $r_{2}-336.75$ & $r_{4}-325$ \\
\hline
\end{tabular}

The two pure equilibria are as predicted in outcomes II and III, and the mixed equilibrium is found at $x=0.888$ and $y=0.8$. The two pure equilibria both result in a new frequency of pollution given by $(1-\delta) p=0.125$. The mixed strategy, however, gives a new frequency of pollution, $(1-x \delta)(1-y \delta) p=0.06678$. Thus the mixed strategy has nearly halved the expected amount of pollution from either pure strategy.

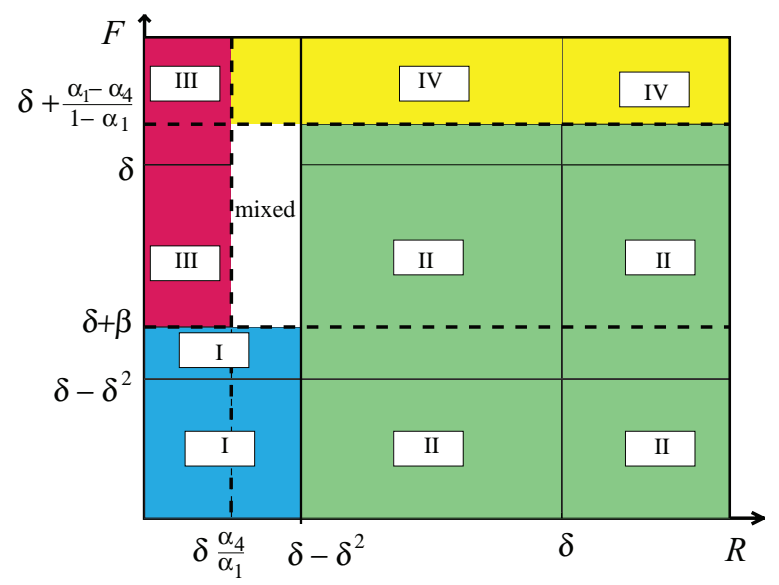

Figure 11. Results of subcase (4b), with $\beta<0$.

\begin{tabular}{|c|c|c|}
\hline Example 5. & Firm controls & Firm does not control \\
\hline \hline $\begin{array}{c}\text { Reg. } \\
\text { controls }\end{array}$ & $f_{1}=481.4875$ & $f_{3}=493.25$ \\
& $r_{1}-275.9875$ & $r_{3}-324.75$ \\
\hline $\begin{array}{c}\text { Reg. does } \\
\text { not control }\end{array}$ & $f_{2}=451.75$ & $f_{4}=425$ \\
& $r_{2}-275.75$ & $r_{4}-325$ \\
\hline
\end{tabular}


Example 5. Here is a final example from subcase (4b) with $\beta>0$ :

$$
\begin{array}{lll}
\text { Given } & \text { Calculated } & \\
P_{F}=1000 & F=0.576 & \beta=0.0919 \\
\Delta=500 & R=0.236 & \delta+\beta=0.4819 \\
C_{F}=120 & & \delta-\delta^{2}=0.2379 \\
C_{R}=29.5 & x=0.6946 & \\
\alpha_{1}=0.5 & y=0.5128 & \delta \frac{\alpha_{3}}{\alpha_{1}}=.234 \\
\alpha_{3}=\alpha_{4} & f_{m}=472.405 & \\
\quad=0.3 & & \\
\begin{array}{l}
\tau=0.4 \\
\delta=0.39
\end{array} & r_{m}=299.744 & \delta+\frac{\alpha_{1}-\alpha_{3}}{1-\alpha_{1}}=0.79 \\
p=0.5 & \\
\text { Since } \delta+\beta \leq F \leq \delta+\frac{\alpha_{1}-\alpha_{3}}{1-\alpha_{1}} \text { and } \delta \frac{\alpha_{3}}{\alpha_{1}} \leq R \leq \\
\delta-\delta^{2}, \text { we predict only one mixed equilibrium: }
\end{array}
$$

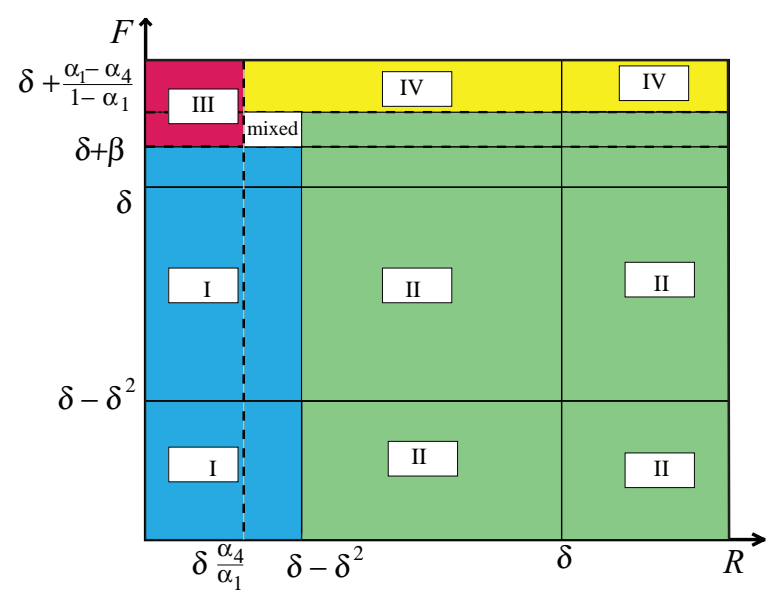

Figure 12. Results of subcase (4b), with $\beta>0$.

\section{Conclusions}

The application of our results would theoretically be to inform policymakers of the likely outcomes of their decisions. The regulator gets to choose the game that will be played and then gets to make the first move. If there is a preferred outcome, especially if it is preferred on the basis of nonmonetary goals, then the policy may be set in such a way as to make that outcome more likely. Here the nonmonetary preferred outcome is usually that of less pollution, since loss of life and extinction of species are difficult to account for in a payoff matrix.

Our results can instruct policymakers at several levels. In an ideal situation, the corporate tax rate and Pigouvian taxes can be adjusted, as in Remarks 1 and 2, to force the game outcomes to have equilibria with low pollution. The real world usually is not ideal, however. Often the policymakers are held financially accountable first and morally accountable second. Of course we are being tongue-in-cheek. It is not simple to separate fiscal efficiency from public welfare.
When the penalties and taxes are fixed by prior factors (perhaps political), it may at first appear that the regulator faces an impossible choice between a financially inferior strategy and a strategy that sacrifices safety or biodiversity for maximizing tax revenue. However, the strength of our analysis is that it can often uncover win-win options: mixed strategies that allow the regulator to choose a policy that is at an equilibrium with less pollution than either pure strategy and higher tax revenue and after-tax profits than in the worstcase pure equilibria for the respective players. In the examples where there were three equilibria, the combined payoffs $f_{m}+r_{m}$ were larger than either $f_{2}+r_{2}$ or $f_{3}+r_{3}$.

Of course "win-win" is a philosophically presumptuous claim. For context we point out that our examples with three equilibria are reminiscent of the classic game of chicken. The two players can either swerve or barrel on straight ahead towards collision. The pure equilibria are when only one player swerves. In our games swerving corresponds to controlling pollution. Thus the interpretation in which $x$ and $y$ are frequencies of control is not so palatable; one head-on crash is more than the public can risk. We prefer the application in which $x$ and $y$ are the relative strengths of two filters applied in tandem.

Importantly, in these mixed equilibria the firm cannot gain from altering its amount of prevention. Thus the government can set its own subsidy of pollution prevention at the mixed equilibria rate. Then it can hope that the firm will follow suit rationally, or at least it can encourage the firm to pay its share on the basis that it has nothing to lose by doing so. In fact, the good public relations gained by the firm will then be a cost-free benefit.

Finally, by examining four cases of penalty policy, we showed that setting the rates of a variable Pigouvian tax can change the game. First, the different subcases have different numbers of possible simultaneous equilibria. Then in Remarks 3, 4, and 5 we point out how the different cases lead to different likelihoods of either or both players controlling pollution.

Further study is warranted by the cases in which there is only one mixed strategy. It has been conjectured that these may harbor semiantagonistic equilibrium points, as defined in [2]. These are points for which neither player's payoff is optimized but for which the environment may reap the benefit. We are thankful to the author of [2] for pointing out this possibility. We also thank Francesco Renna for helpful discussions and advice.

Even more future directions of this research might include taking into account more strategic 


\section{Leading Mathematical Research}

\section{Compositio Mathematica}

publishing first-class mathematical research papers that traditionally focus on

the mainstream of pure mathematics

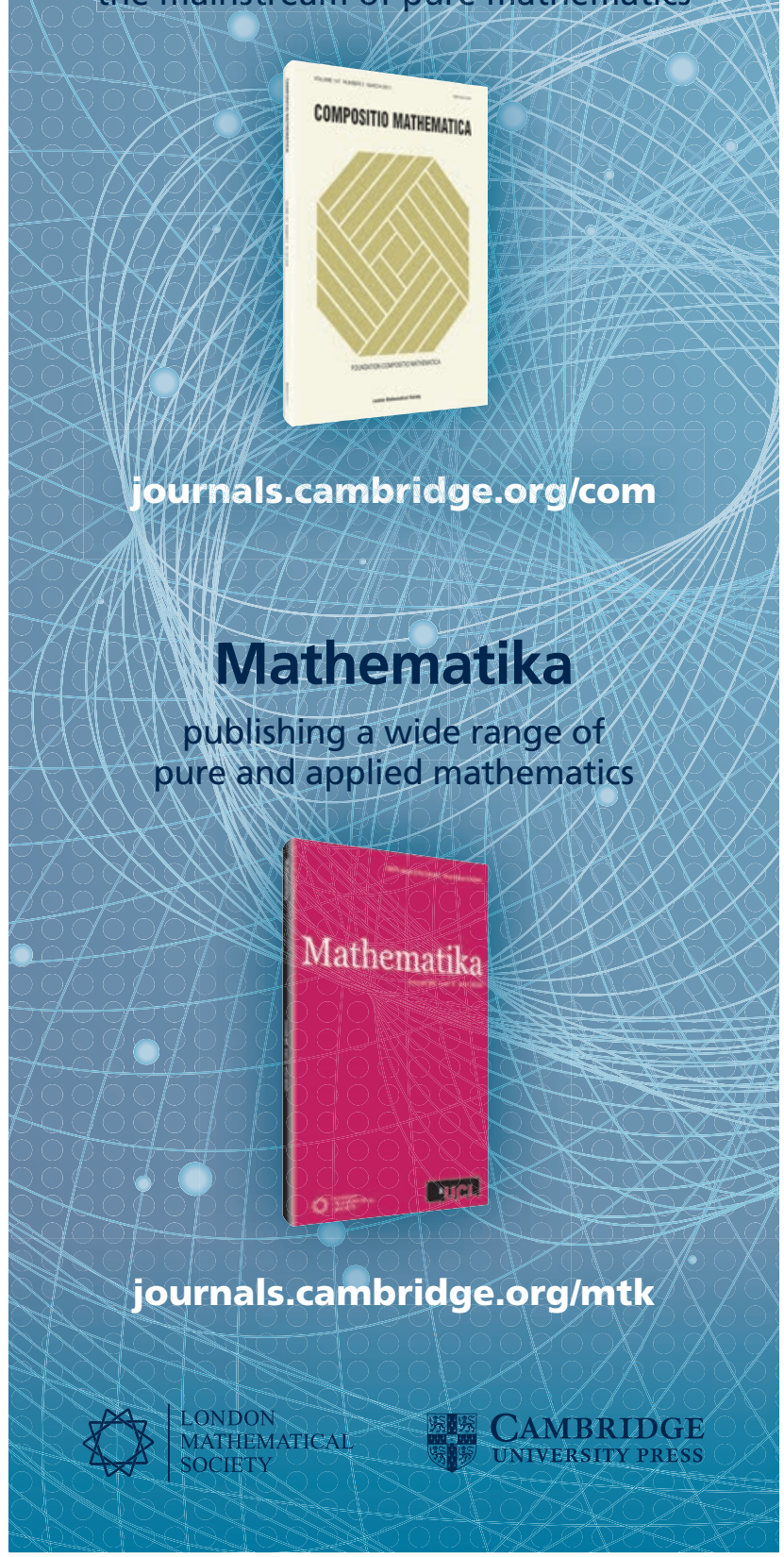

choices for both firm and government. For instance, cases when lobbying by the firm can affect the tax and penalty rates have been studied in [3]. Also, it has been argued that subsidies can be counterproductive if they lead to many more polluting firms entering the market, e.g., in [5]. The model we study here should extend to those more complex situations, where it could again be used to answer the question, when does compromise prevent more pollution?

\section{References}

[1] J. W. Boland, J. Filar, and P. Howlett, Environmental problems, uncertainty and mathematical modeling, Notices of the AMS 57 (2010), 1286-1294.

[2] J. FILAR, Semi-antagonistic equilibrium points and action costs, Cahiers du C.E.R.O. 26 (1984), 227-239.

[3] P. G. FREDRIKSSON, Environmental policy choice: Pollution abatement subsidies, Resource and Energy Economics 20 (1997), 51-63.

[4] Eliza Griswold, The fracturing of Pennsylvania, The New York Times, November 17, 2011.

[5] R. E. KoHN, When subsidies for pollution abatement increase total emissions, Southern Economic Journal 59, No. 1 (1992), 77-87.

[6] J. F. NASH, Non-cooperative games, Annals of Mathematics 54 (1951), 286-295.

[7] C. S. TAPIERO, Environmental quality control and environmental games, Environmental Modelling and Assessment 9 (2004), 201-206.

[8] _ Environmental quality control: A queueing game, Stoch. Environ. Res. Risk Assess. 19 (2005), 59-70. 